\title{
Cat-Scratch Disease in a Child with Ocular Involving: A Case Report
}

\author{
Mirri G1,2, Cavagna $\mathrm{R}^{2}$, Pontari $\mathrm{S}^{2}$, Borrominia $\mathrm{A}^{3}{ }^{*}$, Martinelli $\mathrm{N}^{3}$ and Maggiore $\mathrm{F}^{3}$ \\ ${ }^{1}$ ASST Valle Olona, Department of Pediatric and Neonatology, S. Antonio Abate Hospital-Gallarate, C. Ondoli Hospital-Angera, Italy \\ ${ }^{2}$ ASST Valle Olona, Department of Pediatric and Neonatology, Saronno General Hospital, Italy \\ ${ }^{3}$ Department of Internal Medicine and Medical Therapy, University of Pavia, Medicine and Surgery School, Italy
}

${ }^{*}$ Corresponding author: Andrea Borromini, Department of Internal Medicine and Medical Therapy, University of Pavia, Medicine and Surgery School, Italy, Tel: +39348 887824; E-mail: andrea.borromini01@gmail.com

Received date: February 02, 2019; Accepted date: February 19, 2019; Published date: February 22, 2019

Citation: Mirri G, Cavagna R, Pontari S, Borrominia A, Martinelli N, et al. (2019) Cat-Scratch Disease in a Child with Ocular Involving: A Case Report. Arch Med Vol.11 No.1:3

Copyright: @2019 Mirri G, et al. This is an open-access article distributed under the terms of the Creative Commons Attribution License, which permits unrestricted use, distribution, and reproduction in any medium, provided the original author and source are credited.

\section{Abstract}

We report a case of a 10 -year-old child, who presented with headache, nausea and fever in the past 72 hours. Past medical history was insignificant; parents did not signal any helpful information. Laboratory tests and radiology imaging were normal except for high inflammatory indices and modest spleen enlargement. Rotavirus infection was isolated from the blood cultures.

We want to describe this unusual case of cat-scratch disease presenting with soft mono-lateral optic nerve involvements without the typical scratch related regional lymphadenopathy in order to highlight the heterogeneous presentation of Bartonella Henselae infection, especially in children. Therefore, we should consider cat-scratch disease in any patients with FUO and unspecific systemic symptoms even if there is not a recent cat relate contact and a typical clinical presentation.

\section{Keywords: Child; Cat-scratch disease; Optic nerve}

\section{Introduction}

Cat-scratch disease is usually a self-limited infection caused by the Gram negative bacterial Bartonella henselae. The cat's flea is usually its reservoir and it could deposit the bacterium through the feces on the cat's claws. Human is usually infected by a scratch: Immune-deficient patients and children are at higher risk than others. At the time of primary infection, a macule, papule, or vesicle may form at the site of inoculation followed by regional lymphadenopathy and flu-like symptoms. Incubation period lasts up to 12 days. After 3 weeks, cutaneous signs regress; rarely, hematogenous spread of the initial infection may result in ocular and neurological complications.

\section{Case Report}

The patient, 10-years-old, was admitted to the hospital because of fever during the past three days with peaks of $40^{\circ} \mathrm{C}$, associated with headache and nausea. She got in the emergency room and blood exams results showed a moderate rise of RCP. Further assessments were then necessary. During the hospitalization, general conditions were stable except for sporadic diarrhoea, vomit and fever. Urine cultures, blood cultures, pharyngeal swab, serology for antibodies against Mycoplasma and Chlamydia were carried out but results were in the normal ranges. EKG did not show any abnormality. On the third day, broad spectrum antibiotic therapy was initiated with I.V. cefotaxime because of the persistence of fever. The subsequent blood exams showed a persistent rise of CRP and neutrophil leukocytosis. The second blood culture was still negative. Imaging exams did not reveal any sign about the possible cause of fever. After antibiotic therapy, the patient showed a lowering of the frequency and intensity of body temperature peaks. General conditions improved quickly and inflammation indices decreased. A second line of antibiotic therapy against anaerobic bacteria was initiated and 24-36 hours later the patient showed stable absence of fever. While the patient was recovering, the parents mentioned contacts with a cat during their Easter holidays in Ukraine, so that "cat scratch disease" was immediately suspected. An ophthalmic examination was performed reporting a little optic nerve shade. The patient was discharged in good general conditions, without fever. At the next examination the patient was firmly without fever, although slightly asthenic. Blood serology, still in progress after the discharge, proven positive for Bartonella henselae, which confirmed the suspect of "cat scratch disease". The final diagnosis was infection from Bartonella henselae with mono lateral ocular involving.

\section{Discussion}

Bartonella are facultative intracellular Gram-negative bacteria infecting erythrocytes or endothelial cells [1,2]. $B$ Henselae is especially related to cat scratch disease (CSD) [1,3]. 
Cats are the major host reservoir for $B$. henselae with an antibody prevalence in cat population varying from $5 \%-10 \%$ to $70 \%-80 \%$ depending on geographic area and cat status (domestic or not) [2]. Typical cat scratch disease shows common manifestation: A papule or pustule develops at the scratch site, followed by regional lymphadenopathy, with or without fever, with possible suppuration $[2,4]$.

Ocular manifestations are reported in 5 to $10 \%$ of case 1 : according to the work of Accorint et al. [3], the most frequent ocular manifestation is neuroretinitis which is usually unilateral. The same author states that Bartonellosis can also present as isolated ocular manifestations. Other sings of ocular involvement are Parinaud syndromes, focal retinitis, branch retinal artery and vein occlusions [3,5]. In particular, the usual presentation in children and young adults, is with a wide range of systemic (notably FUO and prolonged fever) and ocular symptoms [3]. Disseminated infection with a vasoproliferative response is typical in immune-compromised individuals [2].

CNS involvement with encephalopathy, seizures, meningitis and meningoencephalitis, and cardiac involvement with Endocarditis (Bartonella accounts for about 3\% of all causes of endocarditis) are less frequent but serious, even life threatening, complications [2]. Among the others, Hepatosplenic, Renal, Hematologic manifestations are possible [2]. We wanted to present this case report to the scientific community in order to increase knowledge about bartonellosis and its manifestations. We believe it is useful to discuss this case for the following reasons: among the complications of bartonellosis, endocarditis and encephalitis have to be searched as soon as suspicions arise, given the serious consenquences and sequelae related.

Bartonella is a significant cause of FUO in children. As shown in a work of Jacobs et al. [6], in which a series of 146 children with fever of unknown origin (FUO) and prolonged fever was analyzed. An established diagnosis was made for $(57.5 \%)$ of patients. The most common infectious disease diagnoses were Epstein-Barr virus infection (15.1\%), osteomyelitis (9.6\%), bartonellosis (4.8\%), and urinary tract infection (4.1\%). Even if our case cannot completely enter in the definition of FUO (but we have to consider the effect of prompt antibiotic therapy on the duration of fever) it has many similar characteristics and suggests considering bartonellosis during differential diagnosis in every case of FUO, particularly in children.
History of contacts with cats, especially with immature cats, is quite a sensitive and simple element to consider, as shown by Massei [7] and Carithers [4] in their respective case series, in which the vast majority of patients reported contact with cats. However, in our case contact with cats was not reported at the beginning, being revealed only after many days. This shows the importance of immediately asking about cats exposition in suspect, or only possible, cases of bartonellosis (fever without evident causes or isolated ocular manifestations in children, endocarditis) [8].

\section{Conclusion}

Many authors have defined bartonellosis a pleomorphic, heterogeneous condition, with both localized and systemic and often unusual-manifestations, presenting a wide range of symptoms. We believe that our case is worth to be added to the existing literature as a further and useful example of this fact.

\section{References}

1. Biancardi AL, Curi AL (2014) Cat-scratch disease. Ocul Immunol Inflamm 22: 148-154.

2. Kalogeropoulos C, Koumpoulis I, Mentis A, Pappa C, Zafeiropoulos P, et al. (2011) Bartonella and intraocular inflammation: A series of cases and review of literature. Clin Ophthalmol 5: 817-829.

3. Accorinti M (2009) Ocular bartonellosis. Int J Med Sci 6: 131-132.

4. Carithers HA (1985) Cat-scratch disease. An overview based on a study of 1,200 patients. Am J Dis Child 139: 1124-1133.

5. Amer R, Tugal-Tutkun I (2017) Ophthalmic manifestations of bartonella infection. Curr Opin Ophthalmol 28: 607-612.

6. Jacobs RF, Schutze GE (1998) Bartonella henselae as a cause of prolonged fever and fever of unknown origin in children. Clin Infect Dis 26: 80-84.

7. Massei F (1999) Bartonella henselae infection in children. Medico e Bambino 18: 410-416.

8. Deschasse C, Bielefeld P, Muselier A, Bour JB, Besancenot JF, et al. (2016) Eye and cat scratch disease: A case series. J Fr Ophtalmol 39: 164-170. 